\title{
The Effects of Binary Stars on the Color-Magnitude Diagrams of Young-Age Massive Star Clusters
}

\author{
Wuming Yang \\ Department of Astronomy, Beijing Normal University,Beijing 100875, China \\ yangwuming@bnu.edu.cn, yangwuming@ynao.ac.cn
}

\begin{abstract}
Extended main-sequence turnoffs (eMSTO) have been observed in the color-magnitude diagram (CMD) of intermediate-age and young star clusters. The origin of the eMSTO phenomenon is still highly debated. Calculations show that the blue and faint (BF) stars in the CMD of NGC 1866 are hydrogen main sequence (MS) + naked He star systems. The He star derives from the massive star of a binary system. The BF stars and the red and faint MSTO stars belong to the same stellar population. The values of $m_{F 336 W}$ and $m_{F 336 W}-m_{F 814 W}$ of the BF stars are mainly determined by the masses of He stars and H-MS stars, respectively. The behaviors of the BF stars in the CMD are well explained by the H-MS + He-star systems. The BF stars provide a strict restriction on the age of the stellar population. Moreover, the bimodal MS of NGC 1866 can also be reproduced by a younger binary population. The calculations show that part of the blue and bright (BB) MS stars of NGC 1866 are H-MS + He-star systems, H-MS + white dwarf systems, and merged stars in a binary scenario. The H-MS stars of the H-MS + He-star systems for the BB stars are significantly more massive than those of the BF stars. Once the H-MS + He-star systems and their membership in NGC 1866 are confirmed, the extended star-formation histories and the effects of binaries can be confirmed in the young star cluster.
\end{abstract}

Subject headings: globular clusters: general — globular clusters: individual (NGC 1866) — Magellanic Clouds - stars: evoluton

\section{INTRODUCTION}

In the classical theory of star formation, a star cluster is considered to be composed of stars belonging to a simple, single stellar population (SSP) with a uniform age and chemical composition. However, the discoveries of double or extended main-sequence turnoffs (eMSTO) (Mackev \& Brobv Nielsen 2007; Glatt et al. 2008; Girardi et al. 2009; Goudfrooij et al. 2009; Milone et al. 2009) in the color-magnitude diagram (CMD) of intermediate-age star clusters in the Large Magellanic Clouds (LMC) are challenging the classical hypothesis. The phenomenon of eMSTOs has been interpreted as meaning that the clusters have experienced extended starformation histories (eSFH) with durations of 100-700 Myr (Glatt et al. 2008; Mackey et al. 2008; Milone et al. 2009; Girardi et al. 2009;
Rubele et al. 2010; Goudfrooij et al. 2011, 2014; Correnti et al 2014). As an alternative interpretation, eMSTOs are thought to be due to coeval populations with different rotation rates (Bastian \& de Mink 2009; Yang et al. 2013; Li et al. 2014; Brandt \& Huang 2015; D'Antona et al. 2015; Niederhofer et al. 2015a) or due to interacting binaries (Yang et al. 2011; Li et al. 2012, 2015). Moreover, stellar variability (Salinas et al. 2016; de Griis 2017) and a variable overshoot of the convective core of stars (Yang \& Tian 2017) may play a potential role in shaping the eMSTO regions as well. The nature of the eMSTO phenomenon is still highly debated.

The problem of the origin of eMSTOs becomes more complicated when they and split main sequences (MS) are found in the CMD of young clusters (less than $\sim 400 \mathrm{Myr}$ ) in the LMC. The 
split MS was first discovered in the CMD of young cluster NGC 1844 (Milone et al. 2013). However, the eMSTOs of young clusters were first observed in NGC 1856 by Correnti et al (2015) and Milone et al. (2015). Young cluster NGC 1856 hosts an eMSTO and a double MS, which changes our understanding of young clusters in the LMC. The eMSTO of NGC 1856 can be interpreted as the superposition of two main populations having the same age but different rotation rates (D'Antona et al. 2015) or as the effects of a variable overshoot of the convective core of stars (Yang \& Tian 2017). Bastian et al. (2017) have inferred the existence of rapidly rotating stars in NGC 1856 and NGC 1850 from H-alpha excess fluxes likely being due to so-called Be candidate stars.

Moreover, eMSTOs and bimodal MS have been observed in young clusters NGC 1755 (Milone et al. 2016), NGC 1850 (Bastian et al. 2016; Correnti et al 2017), and NGC 1866 (Milone et al. 2017). Neither stellar populations with different ages only, nor coeval stellar populations featuring a distribution of stellar rotation rates, properly reproduce the observed split MS and eMSTO (Milone et al. 2017; Correnti et al 2017). eMSTOs were found in young clusters NGC 330, NGC 1805, NGC 1818, and NGC 2164 as well (Li et al. 2017). Li et al. (2017) also show that the observed eMSTOs cannot be explained by stellar rotation alone. Similar cases have been found in intermediate-age star clusters NGC 1987 and NGC 2249, whose eMSTOs cannot be explained solely by a distribution of stellar rotation rates (Goudfrooij et al. 2017). For these clusters, a combination of rotation and an age spread seems to be required to explain observational results (Milone et al. 2016, 2017; Correnti et al 2017; Li et al. 2017; Goudfrooij et al. 2017).

Piatti \& Cole (2017) analyzed the data of young cluster NGC 1971 and found that NGC 1971 exhibits an eMSTO originated mostly by a real age spread. Moreover, Dupree et al (2017) obtained the spectra of 29 eMSTO stars in NGC 1866. The direct spectroscopic measures clearly demonstrate the presence of rapidly rotating stars that are cooler than a population of slowly rotating objects, arguing for an actual spread in age of NGC 1866. However, Lemasle et al. (2017) studied the chemical composition of several Cepheids located in NGC 1866 and found that six Cepheids have a homogeneous chemical composition and are consistent with the red giant branch in the cluster. Their analysis shows that the Cepheids belong to the same stellar population. In line with the comment of Milone et al. (2017) on their observational results of NGC 1866, these observations raise many more questions than they solve.

In addition to the main characteristics of the eMSTO and bimodal MS described by Milone et al. (2017), Figure 1 shows that NGC 1866 has two main MSTOs and that there are many blue and bright (BB) stars and blue and faint $(\mathrm{BF})$ stars in the CMD of NGC 1866. There is a gap between the BB stars and the blue or red MS stars and an upper limit of luminosity for the BF stars; that is, the value of $m_{F 336 W}$ for most of the BF stars is larger than $\sim 21$. An age spread or the effects of rotation cannot produce such BF stars. The behaviors of the BF and BB stars in the CMD might result from binaries. If the $\mathrm{BF}$ and $\mathrm{BB}$ stars are members of NGC 1866, they provide different perspectives on the populations of NGC 1866 and aid us in understanding the nature of the eMSTO phenomenon in young clusters.

In the present work, we mainly focus on whether the characteristics of NGC 1866 can be reproduced by binaries. The paper is organized as follows. Some initial assumptions are given in Section 2, calculation results are shown in Section 3 , and the results are discussed and summarized in Section 4.

\section{STELLAR MODELS AND POPULA- TION SYNTHESIS}

For a binary system, the mass of the primary star, $M_{1}$, is generated in terms of the lognormal initial mass function (IMF) of Chabrier (2001). The mass of the secondary star is then determined by $q M_{1}$, where the $q$ is the ratio of the mass of the secondary to that of the primary and is generated according to an assumed distribution. The distribution of separations $(a)$ between the primary and the secondary stars is assumed to be constant in $\log a$ (Han et al. 1995):

$$
\operatorname{an}(a)=\alpha_{a},
$$

where $\alpha_{a} \approx 0.12328$. The eccentricity $(e)$ of each binary system is assumed to be a uniform distri- 
bution within $0-1$.

In this study, the initial metallicity $Z$ was fixed at 0.008 . Once the initial distributions of the masses $M_{1}$ and $M_{2}\left(q M_{1}\right)$, separation $a$, and eccentricity $e$ were given by Monte Carlo simulation, the sample was evolved to a given age by using the Hurley rapid single/binary evolution codes (Hurley et al. 2000, 2002) to obtain the luminosities and effective temperatures of the stellar population with the given age.

The metallicity $Z$ was converted into $[\mathrm{Fe} / \mathrm{H}]$ by $[\mathrm{Fe} / \mathrm{H}]=\lg (Z / X)-\lg (Z / X)_{\odot} \simeq \lg \left(Z / Z_{\odot}\right)$. There is a bitter controversy between helioseismology and observation about solar metallicity (Yang 2016, and the references therein), but the value of $Z_{\odot}$ in our calculations is 0.02 . The quantities ([Fe/H], $T_{\text {eff }}, \log g, \log L$ ) of evolutionary $\bmod -$ els were then transformed into colors and magnitudes using the color transformation tables of Lejeune et al. (1998). In computing their colors and magnitudes, the binaries with $a \leq 10^{4} R_{\odot}$ were treated as a single point-source object according to the formulas in Zhang et al. (2004). A distance modulus $(m-M)_{0}=18.3$ and reddening $E(B-V)=-0.01$ were adopted in our calculations.

\section{CALCULATION RESULTS}

\subsection{Results of a Uniform $q$ Distribution}

There are about 9900 objects with $15.0 \leq$ $m_{F 336 W} \leq 23.0$ and $-2.0 \leq m_{F 336 W}-m_{F 814 W} \leq$ 1.0 in the observed data of NGC 1866 (Milone et al. 2017). With the assumption that the mass ratio $q$ is a uniform distribution within $0-1$, a sample was evolved to given ages. The CMDs of the simulated populations with different ages are shown in Figure 2. In each panel of Figure 2, there are about 4000 simulated objects with $15.0 \leq m_{F 336 W} \leq$ 23.0 and $-2.0 \leq m_{F 336 W}-m_{F 814 W} \leq 1.0$, where the merged stars make up about $13 \%$. Others are binaries. In our synthesized populations, we included observational errors taken to be a Gaussian distribution with a mean value of 0 and a standard deviation of 0.025 in magnitude and color. The calculations show that the eMSTO and bimodal MS of NGC 1866 cannot be reproduced by the effects of the binaries alone. In order to reproduce the eMSTO region, an age spread of about 150 Myr (from 190 to $340 \mathrm{Myr}$ ) is required in the sim- ulation. The simulation produced a few BF stars and minimal BB stars.

The results cannot exclude the effects of binaries. The mass-ratio $q$ is the key parameter determining the evolutions of binaries, so the calculated results can be affected by the distribution of $q$.

\subsection{Results for Binary Populations with Different $q$ Distributions}

\subsubsection{The binary population reproducing the BF stars}

In order to study whether the BB and BF stars can be reproduced by the evolutions of binaries, we computed the evolutions of a sample of binaries with an uncorrelated $q$. A sample was first generated at random from the IMF as primary stars. Then secondary stars were generated at random from the same IMF. We obtained about 3000 objects with $15.0 \leq m_{F 336 W} \leq 23.0$ and $-2.0 \leq m_{F 336 W}-m_{F 814 W} \leq 1.0$ in each simulation, with the merged stars making up around $13 \%$. The CMDs of the simulated population are shown in Figure 3. For clarity, when the mass of the secondary star evolved to the given age is larger than that of the primary star, the value of the mass ratio is redefined as $1 / q$ in the Figures. The simulations cannot reproduce enough $\mathrm{BB}$ stars, but they can generate some BF stars (see Figure 31), which indicates that the BF stars are relevant to the evolutions of binaries.

The initial value of $q$ of the simulated BF stars is mainly between 3 and 4 , and the values of initial $M_{1}+M_{2}$ of the stars are mainly between about 4 and $5.5 M_{\odot}$ for the population with the age of 340 Myr. Not all binary systems with the special $q$ and initial $M_{1}+M_{2}$ between 4 and $5.5 M_{\odot}$ can evolve into BF stars; only some of the binaries can do so. According to the IMF, the more massive the stars, the smaller their number. If the secondary mass is determined by $q M_{1}$ and the initial $q$ is a uniform distribution within $0-1$, the BF stars could hardly be reproduced in a cluster because there are not enough binary systems with the initial $q$ between 0.25 and 0.33 and $M_{1}+M_{2}$ between 4 and 5.5 $M_{\odot}$. The presence of a large number of BF stars indicates that the initial $q$ for the population of NGC 1866 may follow other distributions or that the BF stars do not belong to NGC 1866.

To reproduce the BF stars, the distribution of 
$q$ is assumed to be

$$
n(q)=\frac{2 q}{\beta},
$$

where $\beta$ is a free parameter. Then mass-ratio $q$ is generated at random by

$$
q=\sqrt{\beta} \times \sqrt{r_{i}}
$$

where $r_{i}$ is a random number within $0-1$. Figure 4 represents the CMDs of simulated populations with different values of $\beta$ but with the same other parameters, which shows that the $\mathrm{BB}$ and BF stars of NGC 1866 are reproduced well by the simulations with $\sqrt{\beta}=4$ or 5 (see panels $b$ and $c$ of Figure 4).

When $\sqrt{\beta} \lesssim 4$, the number of simulated BB and $\mathrm{BF}$ stars increases with the increase in $\sqrt{\beta}$. The smaller the $\sqrt{\beta}$, the redder the produced BF stars. The BF stars of NGC 1866 cannot be reproduced by the simulations with $\sqrt{\beta}<4$. The larger the value of $\sqrt{\beta}$, the more massive the star produced by $q M_{1}$. When the value of $q M_{1}$ is larger than a certain value, the star evolves to a later stage rather than $\mathrm{H}$-MS or He-MS stage at the age of 340 Myr. Thus, when $\sqrt{\beta} \gtrsim 5$, the number of simulated $\mathrm{BB}, \mathrm{BF}$, and $\mathrm{MS}$ stars decreases with an increase in $\sqrt{\beta}$. In order to produce the same number of BF stars, the number of initial models of simulation with $\sqrt{\beta}=6$ is 1.5 times as large as that with $\sqrt{\beta}=5$.

The main difference between a simulated population with $\sqrt{\beta}=4$ and that with $\sqrt{\beta}=5$ is that a simulation with $\sqrt{\beta}=5$ can produce some BF systems with $-1.5 \lesssim m_{F 336 W}-m_{F 814 W} \lesssim-1.0$ but a simulation with $\sqrt{\beta}=4$ cannot produce $\mathrm{BF}$ systems with $-1.5 \lesssim m_{F 336 W}-m_{F 814 W} \lesssim-1.0$ (see Figure 5). Moreover, the number of $\mathrm{BB}$ and MS stars of the population with $\sqrt{\beta}=5$ is slightly lower than that of the population with $\sqrt{\beta}=4$. Furthermore, the simulation with $\sqrt{\beta}=5$ produces a larger number of bright stars that deviate from observation than the simulation with $\sqrt{\beta}=4$ (see panels $c$ and $d$ of Figure 5). The BF stars of NGC 1866 seem to have a gap between $m_{F 336 W}-$ $m_{F 814 W} \approx-1.0$ and $m_{F 336 W}-m_{F 814 W} \approx-1.4$ (see panel $a$ of Figure 5), which could be used to limit the value of $\beta$.

Fixing the value of $\sqrt{\beta}$ at 4 , we computed stellar populations with different ages. The results are represented in Figure 6. The calculations show that the BF stars and the red and faint MSTO of NGC 1866 are reproduced well by the populations with an age between about 320 and 340 Myr. The BF stars and the red and faint MSTO belong to the same population. There are almost no BF stars with $m_{F 336 W}<\sim 21$ for the stellar populations (see panels $c$ and $d$ of Figure 6). A large number of BB stars were reproduced by the calculations as well.

\subsubsection{The blue and faint stars}

For both the simulated and observed populations, there are only a few BF stars brighter than $m_{F 336 W} \sim 21$ (see Figure 5 and panels $c$ and $d$ of Figure 6], which indicates that the luminosity of $m_{F 336 W}$ for BF stars with the given age has an upper limit. Our calculations show that a BF star is a binary system consisting of a hydrogen MS star and a naked helium star. The initial value of $q$ of the system is mainly between about 2.5 and 4 . The He stars evolve from the massive stars of the systems. The initial masses of the massive stars are mainly in the range of $\sim 3.0-3.5 M_{\odot}$ for the population of the age of $340 \mathrm{Myr}$. More massive stars have evolved into later phases, but lower mass stars have not yet evolved into He stars.

The masses of the He stars of BF systems are mainly between about 0.45 and $0.54 M_{\odot}$, but the masses of the H-MS stars are mainly in the range of $\sim 0.7-1.3 M_{\odot}$. The value of $m_{F 336 W}$ of a $\mathrm{BF}$ system is mainly dependent on its He star because the value of $m_{F 336 W}$ of its H-MS star is much larger than that of the He star when the mass of the H-MS star is lower than $1.4 M_{\odot}$ (see Figure 7). The luminosity of a He star is determined by its mass. The value of $m_{F 336 W}$ of the He-MS stars with masses in the range of $\sim 0.50-0.54 M_{\odot}$ is around 21. More massive He stars have evolved into the Hertzsprung Gap or later phases. The lifetimes of the phases are very short, which leads to the fact that there are only a few BF stars with $m_{F 336 W}<\sim 21$ in our simulations. Therefore, the $\mathrm{BF}$ candidates cannot blend with BB stars in the CMD unless the number of H-MS + He-star systems is large enough to contain many more massive He stars at the given age.

The luminosities of $m_{F 814 W}$ of the BF systems are mainly dependent on the H-MS stars of the systems because the values of $m_{F 814 W}$ of the H- 
MS stars are much lower than those of the He stars (see Figure 7). But the values of $m_{F 336 W}$ of the systems only slightly decrease with an increase in mass of the H-MS stars. Thus the variation in mass of the H-MS stars mainly affects the values of $m_{F 336 W}-m_{F 814 W}$. For the BF stars, the more massive the H-MS stars, the lower the values of $m_{F 814 W}$, and the larger the values of $m_{F 336 W}-$ $m_{F 814 W}$; but the more massive the He stars, the lower the values of $m_{F 336 W}$. The characteristics of the BF stars of NGC 1866 are consistent with those of H-MS + He-star systems (see Figure 8).

However, when the H-MS star is massive than about $1.4 M_{\odot}$, the luminosity of the H-MS + Hestar system can be significantly affected by the H-MS star. When the H-MS star is massive than about $1.5 M_{\odot}$, the values of $m_{F 336 W}$ and $m_{F 814 W}$ of the system are mainly determined by the H-MS star. The system appears as a MS star rather than a BF star. As a consequence, there is an upper limit of $m_{F 336 W}$ for BF stars with the age of 340 Myr at $m_{F 336 W} \sim 21$. The increase in mass of the H-MS stars of BF systems cannot lead to the fact that $\mathrm{BF}$ candidates blend with the $\mathrm{BB}$ stars in the CMD. The BF stars with an approximate $m_{F 336 W}$ are almost in line in the CMD due to the difference in mass of H-MS stars (see panels $c$ and $d$ of Figure 6). These characteristics can be used to restrict the age of young star clusters.

If the age of the BF stars is younger than about 320 - $340 \mathrm{Myr}$, the value of the upper limit of $m_{F 336 W}$ will be smaller than 21 due to the presence of more massive He stars (see panel $a$ of Figure 6). In the observed sample, there are only several BF stars with $m_{F 336 W}$ between about 20 and 21, but a large number between around 21 and 23. Both the BF stars and the red and faint MSTO stars belong to the same population. This indicates that there is a stellar population as old as $~ 320-340$ Myr in NGC 1866. Moreover, BF stars can only derive from binary systems with an initial mass ratio in a narrow range. Thus there should be many binaries in NGC 1866.

The orange dots in the lower-left corners of the panels of Figure 8 represent He-star + white dwarf (WD) systems. The magnitudes of these systems are almost entirely determined by their He stars. Thus they look like a single He star and have an approximately equal $m_{F 336 W}-m_{F 814 W}$ or $m_{F 555 W}-m_{F 814 W}$. Figure 7 shows that the longer the effective wavelength or the more massive the $\mathrm{H}-\mathrm{MS}$ star, the more easily is the magnitude of $\mathrm{H}$ MS + He-star system affected by the H-MS star. Therefore, the H-MS + He-star systems are more easily separated from MS by $m_{F 336 W}-m_{F 814 W}$ rather than by $m_{F 555 W}-m_{F 814 W}$. The lower the mass of a H-MS star, the smaller is the contribution of the H-MS star to the $m_{F 336 W}$ and $m_{F 555 W}$ of the H-MS + He-star system; so the BF systems with lower-mass H-MS stars are closer to WD + He-star systems in $m_{F 336 W}-m_{F 555 W}$ (see panel $c$ of Figure 8). This indicates that BF systems are more easily displayed on $m_{F 336 W}-m_{F x x x W}$, where the $x x x$ represents the effective wavelength of other filters, such as 450, 555, or 814. It indicates, too, that BF systems have different behaviors in different colors, which can aid us in confirming the BF systems.

The simulation with a larger $\beta$ can produce the $\mathrm{H}-\mathrm{MS}+\mathrm{He}-\mathrm{star}$ systems with a lower-mass H-MS star, i.e. bluer BF stars when $\sqrt{\beta} \leq 5$, which leads to the fact that simulations with $\sqrt{\beta}=4$ cannot produce the BF stars with $m_{F 336 W}-m_{F 814 W} \lesssim$ -1.0 .

\subsubsection{The blue and bright stars}

The simulations also produce many BB stars that are mainly merged stars, H-MS + He-star systems, and H-MS + WD systems. Such stars are also called blue stragglers (Strom \& Strom 1970; Pols \& Marinus 1994). The H-MS stars had accreted mass from their companions. The value of initial $q$ of the systems is mainly in the range of around 1.5 - 2.5. As with BF stars, the initial masses of the massive stars that evolved into the naked He-MS stars are mainly between about 3.0 and $3.5 M_{\odot}$; but the initial masses of the $\mathrm{H}$ MS stars are higher than those of BF systems. The masses of the H-MS stars of BB systems are mainly between about 2.5 and $4 M_{\odot}$, which are obviously more massive than those of BF systems. The luminosities of the BB systems are more dependent on their H-MS stars than on their $\mathrm{He}$ stars. The BB stars thus look like MS stars and are more luminous than BF systems.

The accretion and merging make the stars bluer and brighter than the MSTO stars. The value of mass accretion rates can affect the luminosity of stars, but the mass accretion rate and the process of merging are not definitely known in the theory 
of stellar evolution. The more the mass accreted by a star, the larger is the star's luminosity. The uncertainty of the rate could give rise to the fact that the simulated $\mathrm{BB}$ stars are more scattered than the observed ones in the CMD (see Figures 6) and (8).

The contribution of the He stars to the $m_{F 336 W}$ of the systems is larger than to the $m_{F 814 W}$ of the systems. The radius of the He stars is of the order of $0.1 R_{\odot}$. The He stars of the systems could be outshone by their companions or disks and be difficult to observe. This may lead to the fact that the observed BB stars are slightly fainter and redder than the theoretical models in the CMD.

Some BB stars are H-MS + WD systems. The masses of the H-MS stars are mainly between about 3 and $4 M_{\odot}$, but those of the WDs are mainly in the range of $\sim 0.55-0.7 M_{\odot}$. The luminosities of the systems are mainly determined by the H-MS stars. Thus they look like blue MS stars rather than WDs.

\subsubsection{The blue $M S$}

Panel $a$ of Figure 6 shows that the simulated stellar population with the $q$ distribution and age of 190 Myr cannot reproduce the blue MS of NGC 1866. Moreover, the population contains many BF stars with $m_{F 336 W}$ between about 20 and 21 . The absence of $\mathrm{BF}$ stars with $m_{F 336 W}$ between about 20 and 21 in the observed sample indicates that properties of the blue MS stars of NGC 1866 should be different from those of the simulated population.

We computed the evolutions of binary populations with a Gaussian mass-ratio distribution. The mean value and standard deviation of the Gaussian distribution is 0.6 and 0.11 , respectively. Figures 9 and 10 show that the bimodal MS and blue MS of NGC 1866 can be reproduced by the binary population with an age of about 190 Myr. The blue stragglers with $m_{F 336 W} \sim 16$ and $m_{F 336 W}-m_{F 814 W}<-1.0$ of NGC 1866 are reproduced as well. As we have just said, however, the BF stars do not appear in this simulation; so this young population is not incompatible with the constraints of the observed BF stars.

The blue MS is mainly composed of merged stars and binaries with a $q$ less than about 0.5 (see Figure 11). Most of the simulated systems have an initial $q$ larger than 0.5 , which leads to the fact that the red MS is denser than the blue MS. Mass accretion and merging can lead to the fact that the number of stars with masses in a certain range can increase, but those with masses in another range decreases ( see Figure 11). This results in the fact that the blue MS is discontinuous in the CMD.

The simulated blue stragglers are composed of merged MS stars and H-MS + He-star systems. The masses of the He-MS stars are mainly between about 0.6 and $0.7 M_{\odot}$. As we have noted, the $\mathrm{H}$ MS stars had accreted mass from their companions. The masses of these stars and the merged MS stars are mainly in the range of $\sim 5.0$ and 6.5 $M_{\odot}$, which is much larger than the masses of the MSTO stars of the SSP with the age of 190 Myr. The luminosity of the H-MS + He-star system is mainly dependent on the H-MS star rather than the He star. Thus the system is a blue and bright star rather than a blue and faint star.

Moreover, part of the simulated blue MS $\left(m_{F 336 W}<19\right)$ are merged MS stars, H-MS + He-star systems, and H-MS + WD systems. The masses of the He-MS stars are mainly between about 0.6 and $0.7 M_{\odot}$; but those of the WDs mainly between the range of $\sim 0.9-1.0 M_{\odot}$. For this young stellar population, the masses of the He stars of H-MS + He-star systems are mainly between about 0.6 and $0.7 M_{\odot}$, which are larger than those of the stellar population with the age of $340 \mathrm{Myr}$.

Figure 12 presents the CMD of simulated multiple populations that are the mixture of the binary population characterized by Gaussian massratio distribution and an age of $190 \mathrm{Myr}$ with the population with $\sqrt{\beta}=5$ and an age of $340 \mathrm{Myr}$. It shows that the blue and the red MS gradually merge when the value of $m_{F 336 W}$ is larger than 20 . When the value of $m_{F 336 W}$ is less than 20 , however, the separation between the blue and the red MS is almost not affected by the old population.

\subsubsection{Other Clusters}

The CMDs of NGC 1806 and NGC 1856 have been given by (Milone et al. 2009, 2015). There are almost no BF stars in the observed CMDs. With the same initial parameters and $\sqrt{\beta}=5$, we computed stellar populations with different ages. 
The blue stragglers and the blue and bright MSTO of NGC 1806 can be reproduced by a population with the age of 1.35 Gyr (see Figure13); but there are only a few BF stars in the simulated population. The faint and red MSTO of NGC 1856 can be reproduced by a population with the age of 600 Myr, and the few BF stars of NGC 1856 also are reproduced by the simulated population. The observations and simulations show that BF stars are correlated with the age of clusters. They appear more easily in a young cluster.

\section{DISCUSSION AND SUMMARY}

\subsection{Discussion}

We noticed that the ages of binary populations are larger than those given by Milone et al. (2017), which may be due to the effects of binaries. Similar to Milone et al. (2017), we obtained an age of about 150 Myr for NGC 1866 when we used a SSP to fit the blue MS of NGC 1866 (see Figure 14). Moreover, the simulated age of a cluster is related to $[\mathrm{Fe} / \mathrm{H}]$. The uncertainty of $[\mathrm{Fe} / \mathrm{H}]$ can affect the age.

Assuming a constant mass-ratio distribution, Milone et al. (2017) found that the fraction of binaries in NGC 1866 is about 0.28 . In our simulations, in the primary stage of evolutions all models are members of binaries. When the models are evolved to the age of 190 or $340 \mathrm{Myr}$, the merged stars make up only about $12 \%-14 \%$ of the obtained objects; but all the others are still binaries. Moreover, all the simulated BF stars are binaries. The ratio of merged He stars to He-star + WD systems in Figures 8 and 15 is around $0.08-0.12$. Our binary fraction is much larger than that given by Milone et al. (2017).

The IMF does not affect our results. Basing on Salpeter (1955) IMF, we obtained almost the same results (see Figure 15). The difference between the results obtained from Salpeter (1955) IMF and those based on Chabrier (2001) IMF can be neglected. The value of $E(B-V)$ is -0.01 for NGC 1866, 0.08 for NGC 1856, and 0.16 for NGC 1806 , which may be related to the mixing-length parameter. That parameter is unadjustable in the Hurley et al. (2002) codes and is calibrated to a solar model. The larger the parameter, the smaller the radius of stellar models, and the higher their effective temperature. If the value of the mixing- length parameter increases with an increase in the mass of stars but is fixed at the value calibrated to a low-mass star, one could find that for clusters with the same reddening the value of theoretical $E(B-V)$ decreases with a decrease in age of the clusters because the masses of MSTO stars increase with the decrease in age of the clusters.

The rotation velocity of merged stars is hard to estimate. If they lost angular momentum as they merged, they are slowly rotating stars; but if their angular momentum was conservative, they are fast rotators. In order to distinguish the merged stars from binaries in Figure 16, their velocities are assumed to be $150 \mathrm{~km} \mathrm{~s}^{-1}$. We calculated rotational velocities and orbital velocities with the assumption that the rotation rate of a star is equal to the revolution rate of the binary system. The rotational and orbital velocities of many stars are of the order of 100 and $200 \mathrm{~km} \mathrm{~s}^{-1}$, respectively; but orbital velocities can be as high as about 300 $\mathrm{km} \mathrm{s}^{-1}$. The fraction of MSTO stars with orbital velocities higher than $200 \mathrm{~km} \mathrm{~s}^{-1}$ in the old population is larger than that in the young population (see panels $b$ and $d$ of Figure 16).

The eMSTO and bimodal MS of NGC 1866 can be explained by a combination of rotation and age spread (Milone et al. 2017; Correnti et al 2017); but the eMSTO can also be explained by the effects of a large overshoot of the convective core of stars (Yang \& Tian 2017). A single He star, WD, or MS star cannot appear as a BF star, BB star, or blue straggler. Thus the H-MS + He-star systems and H-MS + WD systems are the main characteristics that can distinguish the binary scenario from the rotation scenario and the overshoot scenario.

The calculations show that the BF stars are $\mathrm{H}-$ MS + He-star systems. The H-MS star is a low mass star, and it hardly accretes mass from its companion. The BF stars and the red and faint MSTO belong to the same population. The BF stars can provide a constraint on the age of the stellar population. Once the BF stars are determined to be the members of the cluster, the existence of an old stellar population in the cluster will be confirmed. The initial $q$ of the H-MS + Hestar systems is in a narrow range. The observed $\mathrm{BF}$ stars could be used to estimate the fraction of binaries in the cluster.

The simulated bimodal MS is sensitively dependent on the mean value of the Gaussian mass-ratio 
(q) distribution and can be reproduced when the mean value is in the range of about $0.6-0.67$.

For a population with an age of $340 \mathrm{Myr}$, the masses of He stars and WDs are mainly within the range of $\sim 0.46-0.54$ and $\sim 0.55-0.7 M_{\odot}$, respectively; but for that with an age of $190 \mathrm{Myr}$, their masses are mainly within the range of $\sim 0.6-0.7$ and $\sim 0.9-1.0 M_{\odot}$, respectively. The fundamental parameters of binary stars are more easily determined from light curves than those of single stars. If the young cluster consists of two main populations, there would be a bimodal distribution of the mass of He stars.

Furthermore, part of the blue stragglers are massive H-MS + He-star systems. If they derive from a young population with an age of about 190 Myr, the masses of the He stars are between about 0.6 and $0.7 M_{\odot}$, but those of the H-MS stars are between around 5 and $6.5 M_{\odot}$. The masses of the H-MS and He stars of the BF systems of the stellar sub-population with the age of $340 \mathrm{Myr}$ are virtually all lower than 1.4 and $0.54 M_{\odot}$, respectively. If one can determine that part of the blue stragglers are the massive H-MS + He-star systems and that the BF stars are the low mass H-MS + Hestar systems, that will confirm the existence of the $\mathrm{eSFH}$ and the role of binaries.

Photometric errors for bright MS stars are around $0.02 \mathrm{mag}$ in color (Correnti et al 2014; Milone et al. 2016). The errors could increase with an increase in magnitude. The characteristics of the BF population are mainly dependent on He stars. The values of $m_{F 336 W}-m_{F 814 W}$ of the BF population are mainly between about -2.0 and -0.3 , i.e. the change of $\left|m_{F 336 W}-m_{F 814 W}\right|$ is large than 0.1 . Even photometric errors increase from 0.02 to $0.1 \mathrm{mag}$, our results for the BF population are not changed (see Figure 17); but the simulated MS broadens.

\subsection{Summary}

In this work, we calculated different binary populations. The calculations show that the BF stars of NGC 1866 are H-MS + He-star systems, which derive from the evolutions of binaries with the initial $q$ in the range of about $2.5-4$. The He star comes into being from the evolution of the massive star of the system. The value of $m_{F 336 W}$ of a BF star is mainly dependent on the mass of the He star. The mass of most of the He stars is lower than $0.54 M_{\odot}$ for the population with the age of 340 Myr, which leads to the fact that the $m_{F 336 W}$ of most of the BF stars is larger than $\sim 21$. The value of $m_{F 336 W}-m_{F 814 W}$ of a $\mathrm{BF}$ star mainly depends on the mass of the H-MS star that lies mainly within the range of about $0.7-1.3 M_{\odot}$. The more massive the H-MS star, the larger the value of $m_{F 336 W}-m_{F 814 W}$ for a BF star. However, when the mass of the H-MS star is larger than $1.5 M_{\odot}$, the system looks like an MS star rather than a $\mathrm{BF}$ star. If the population of $\mathrm{BF}$ stars can be confirmed to be members of NGC 1866, this would directly show that NGC 1866 hosts a population older than the blue MS, otherwise a mechanism making some stars bluer and brighter, such as variable overshoot, is required to explain the blue MS.

The bimodal MS of NGC 1866 can be produced by the binary population with the Gaussian $q$ distribution and the age of $190 \mathrm{Myr}$. The calculations show that the BB stars of NGC 1866 are mainly merged stars, H-MS + He-star systems, and H-MS $+\mathrm{WD}$ systems, which leads to the discontinuities between the BB stars and the blue MS. The H-MS + He-star systems and H-MS + WD systems are the main characteristic of the binary models, and this can be used to confirm or exclude the binary scenario.

Moreover, the calculations show that the blue stragglers of NGC 1866 and the blue MS belong to the same population. The blue stragglers consist of the merged stars and H-MS + He-star systems. The masses of the He stars are mainly between about 0.6 and $0.7 M_{\odot}$, which are larger than those of the BF stars. Therefore, the existence of the blue stragglers and the BF stars can confirm the existence of the eSFH.

To explain the eMSTO and bimodal MS of NGC 1866, a combination of an age spread and binary population is required. The role of binaries in the formation of the eMSTO and bimodal MS can be tested by whether part of the BB stars are H-MS + He-star systems or H-MS + WD systems. Moreover, the eSFH can be confirmed by whether the BF stars are members of NGC 1866, because the $\mathrm{BF}$ stars belong to an older population in the theoretical model. 
The author thanks the anonymous referee for helpful comments that helped the author improve this work, A. P. Milone for providing the observed data of NGC 1866, and Daniel Kister for help in improving the English, and acknowledges the support from the NSFC 11773005, U1631236, and 11273012 .

\section{REFERENCES}

Bastian, N., Cabrera-Ziri, I., Niederhofer, F., et al. 2017, MNRAS, 465, 4795

Bastian, N., \& de Mink, S. E. 2009, MNRAS, 398, L11

Bastian, N., Niederhofer, F., Kozhurina-Platais, V., et al. 2016, MNRAS, 460, L20

Brandt, T. D., Huang, C. X. 2015, ApJ, 807, 25

Chabrier, G. 2001, ApJ, 554, 1274

Correnti, M., Goudfrooij, P., Kalirai, J. S., et al. 2014, ApJ, 793, 121

Correnti, M., Goudfrooij, P., Puzia, T. H., de Mink, S. E. 2015, MNRAS, 450, 3054

Correnti, M., Goudfrooij, P., Bellini, A., Kalirai, J. S. Puzia, T. H. 2017, MNRAS, 467, 3628

D'Antona, F., Di Criscienzo, M., Decressin, T., et al. 2015, MNRAS, 453, 2637

de Grijs, R. 2017, NatAs., 1, 0011

Dupree, A. K., Dotter, A., Johnson, C. I., et al. 2017, ApJL, 846, L1

Girardi, L., Rubele, S., \& Kerber, L. 2009, MNRAS, 394, L74

Glatt, K., Grebel, E. K., \& Sabbi, E. et al. 2008, AJ, 136, 1703

Goudfrooij, P., Girardi, L., Kozhurina-Platais, V., et al. 2014, ApJ, 797, 35

Goudfrooij, P., Puzia, T. H., Chandar, R., \& Kozhurina-Platais, V. 2011, ApJ, 737, 4

Goudfrooij, P., Puzia, T. H., Kozhurina-Platais, V., \& Chandar, R. 2009, AJ, 137, 4988

Goudfrooij, P., Girardi, L., Correnti, M. 2017, ApJ, 846, 22
Han, Z., Podsiadlowski, P., \& Eggleton, P. P. 1995, MNRAS, 272, 800

Hurley, J. R., Pols, O. R., \& Tout, C. A. 2000, MNRAS, 315, 543

Hurley, J. R., Tout, C. A., \& Pols, O. R. 2002, MNRAS, 329, 897

Lejeune, T., Cuisinier, F., \& Buser, R. 1998, A\&A, 130,65

Lemasle, B., Groenewegen, M. A. T., Grebel, E. K., et al. 2017, A\&A, 608, A85

Li, C., de Grijs, R., Deng, L. 2014, Nature, 516, 367

Li, C., de Grijs, R., Deng, L., \& Milone, A. P. 2017, ApJ, 844, 119

Li, Z., Mao, C., Chen, L. 2015, ApJ, 802, 44

Li, Z., Mao, C., Chen, L., Zhang, Q. 2012, ApJ, 761,22

Mackey, A. D., \& Broby Nielsen, P. 2007, MNRAS, 379, 151

Mackey, A. D., Broby Nielsen, P., Ferguson, A. M. N., \& Richardson, J. C. 2008, ApJ, 681, L17

Milone, A. P., Bedin, L. R., Piotto, G., \& Anderson, J. 2009, A\&A, 497, 755

Milone, A. P., Bedin, L. R., Cassisi, S., Piotto, G., Anderson, J., Pietrinferni, A., Buonanno, R. 2013, A\&A, 555, A143

Milone, A. P., Bedin, L. R., Piotto, G., et al. 2015, MNRAS, 450, 3750

Milone, A. P., Marino, A. F., D'Antona, F., et al. 2016, MNRAS, 458, 4368

Milone, A. P., Marino, A. F., D'Antona, F., et al. 2017, MNRAS, 465, 4363

Niederhofer, F., Georgy, C., Bastian, N., Ekstrm, S. 2015a, MNRAS, 453, 2070

Piatti, A. E., \& Cole, A. 2017, MNRAS, 470, L77

Pols, O. R., \& Marinus, M. 1994, A\&A, 288, 475

Rubele, S., Kerber, L., \& Girardi, L. 2010, MNRAS, 403, 1156 
Salinas, R., Pajkos, M. A., Strader, J., Vivas, A. K., Contreras Ramos, R. 2016, ApJL, 832, L14

Salpeter, E. E. 1955, ApJ, 121, 161

Strom, K. M., \& Strom, S. E. 1970, ApJ, 162, 523

Yang, W. 2016, ApJ, 821, 108

Yang, W. M., Bi, S. L., Meng, X. C., Liu, Z. 2013, ApJ, 776, 112

Yang, W. M., Meng, X. C., Bi, S. L., et al. 2011, ApJ, 731, L37

Yang, W., \& Tian, Z. 2017, ApJ, 836, 102

Zhang, F., Han, Z., Li, L., Hurley, J. R. 2004, A\&A, 414, 117

This 2-column preprint was prepared with the AAS LATEX

macros v5.2. 

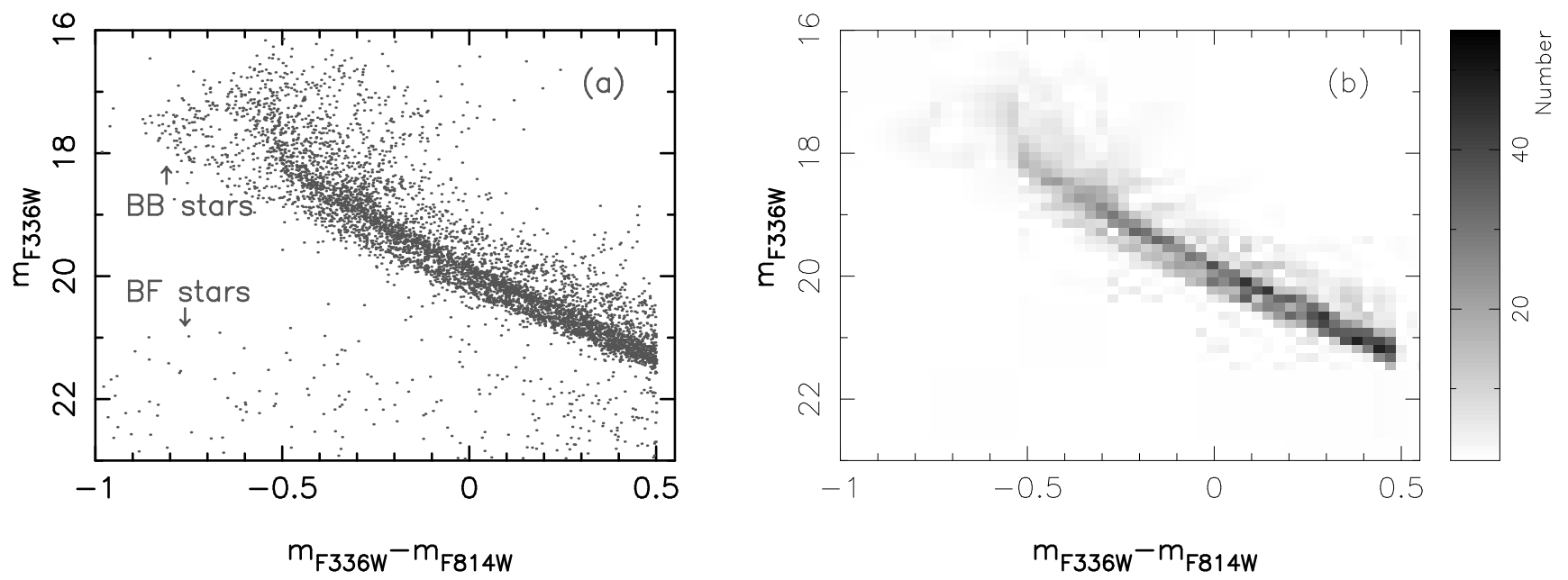

Fig. 1. - The color-magnitude diagrams of NGC 1866 obtained by Milone et al. (2017). The blue and bright stars on the top left of panel $a$ are labelled as BB stars, while the blue and faint stars on the bottom left of panel $a$ are labelled as BF stars. The grey scale in panel $b$ is proportional to the number of stars. 

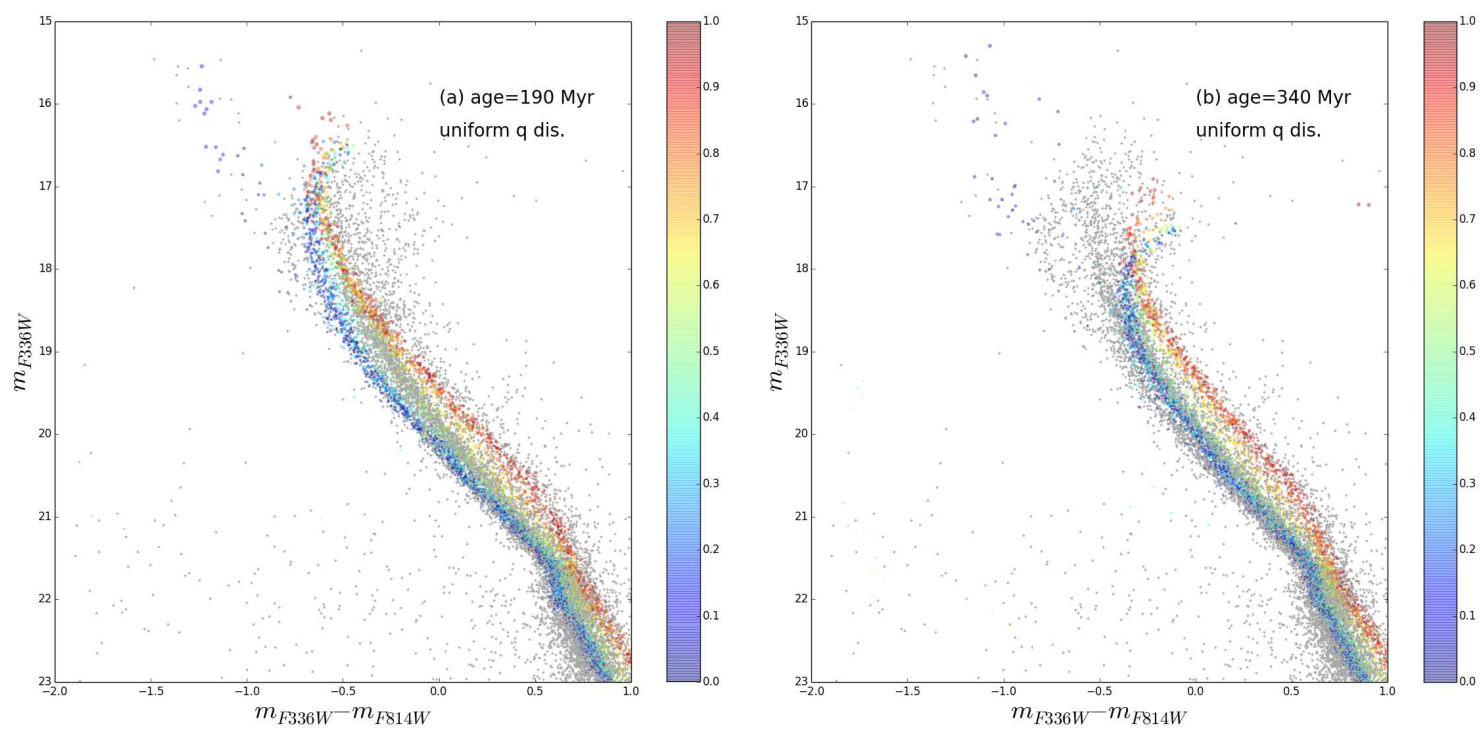

Fig. 2.- CMDs of the observed and simulated stellar populations. The grey dots refer to the observed data of NGC 1866 (Milone et al. 2017). The color dots represent simulated stellar populations. The size and the color of the color dots are proportional to stellar mass and mass-ratio $q$, respectively. The value of $q$ of merged stars is 0 . 

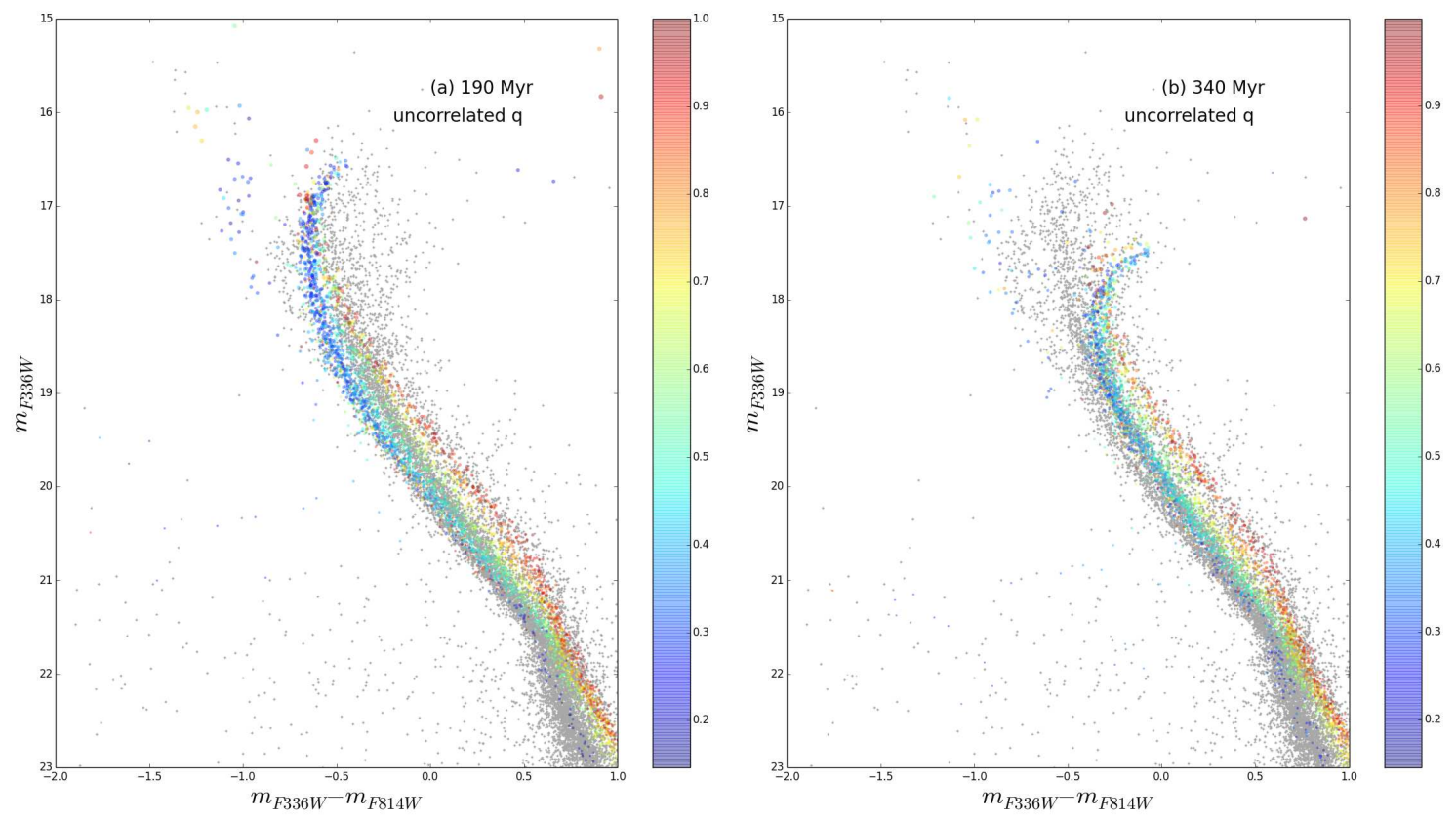

Fig. 3.- Same as Figure 2 but the initial mass of the secondary is uncorrelated with that of the primary. In the figure, when the mass of the secondary is larger than that of the primary, the value of mass ratio is redefined as $1 / q$. Thus the value of mass ratio keeps within $0-1$ in the figure. 

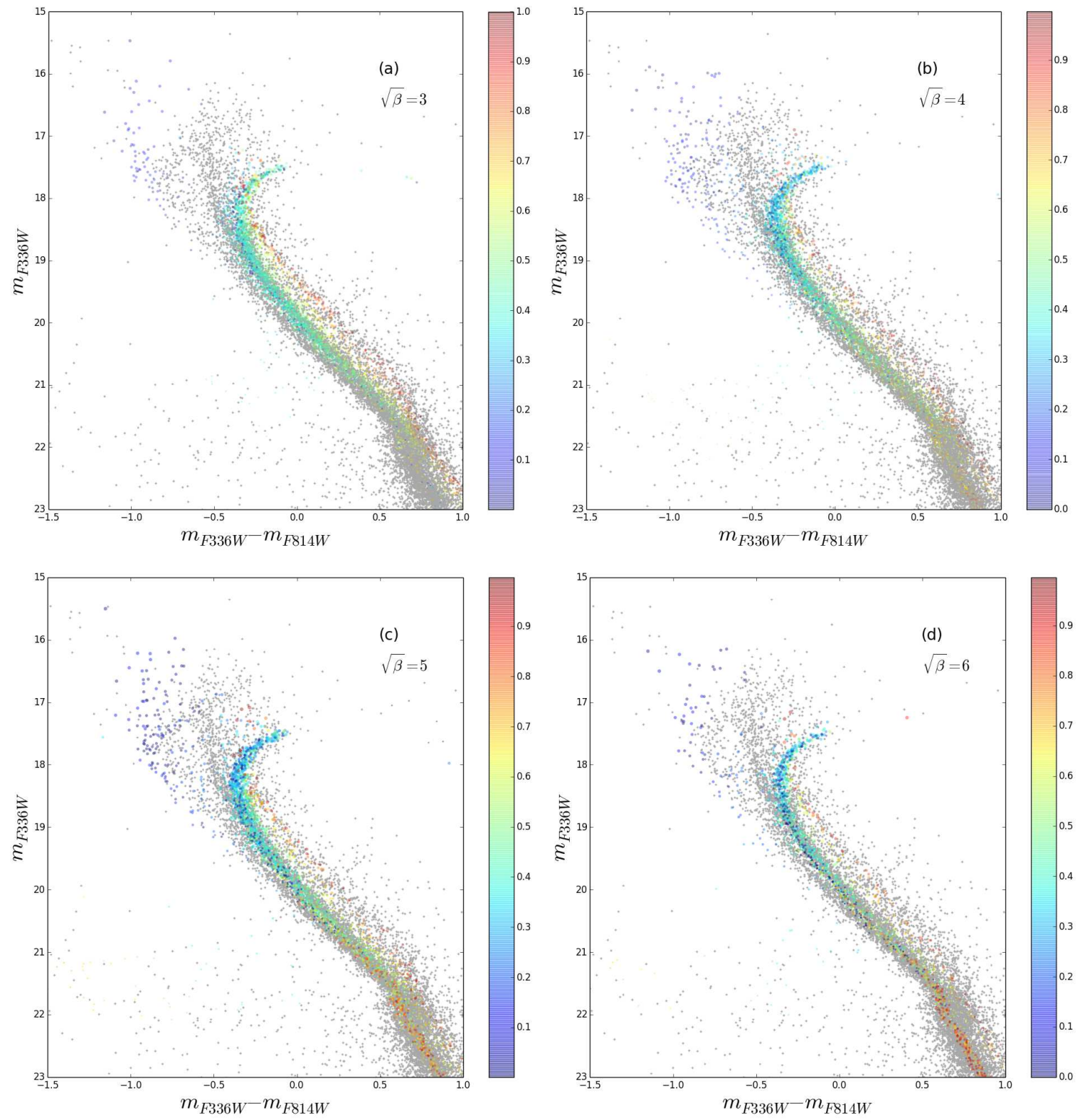

Fig. 4.- CMDs of simulated stars with the age of $340 \mathrm{Myr}$ but with different $\beta$. There are about 3000 simulated objects in panels $a$ and $b, 2500$ objects in panel $c, 1600$ objects in panel $d$. The merged stars make up about $13 \%$. When the mass of the secondary is larger than that of the primary, the value of mass ratio is redefined as $1 / q$. The larger the $\beta$, the larger the number of massive stars. Thus the number of the simulated population decreases. 

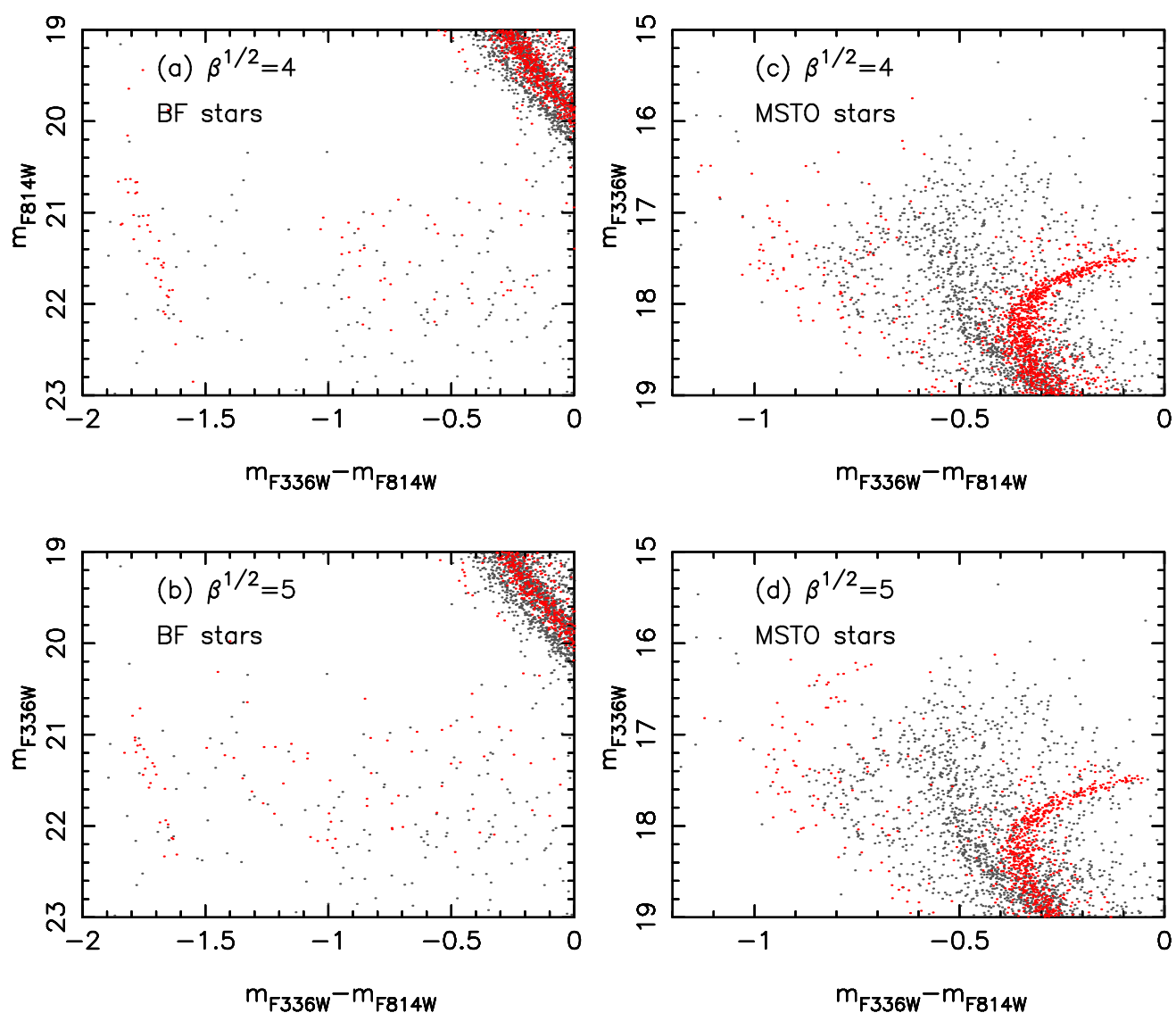

Fig. 5.- CMDs of simulated populations with the age of $340 \mathrm{Myr}$ but with different $\beta$. The grey dots refer to the data of NGC 1866 . The red dots represent the simulated populations. 

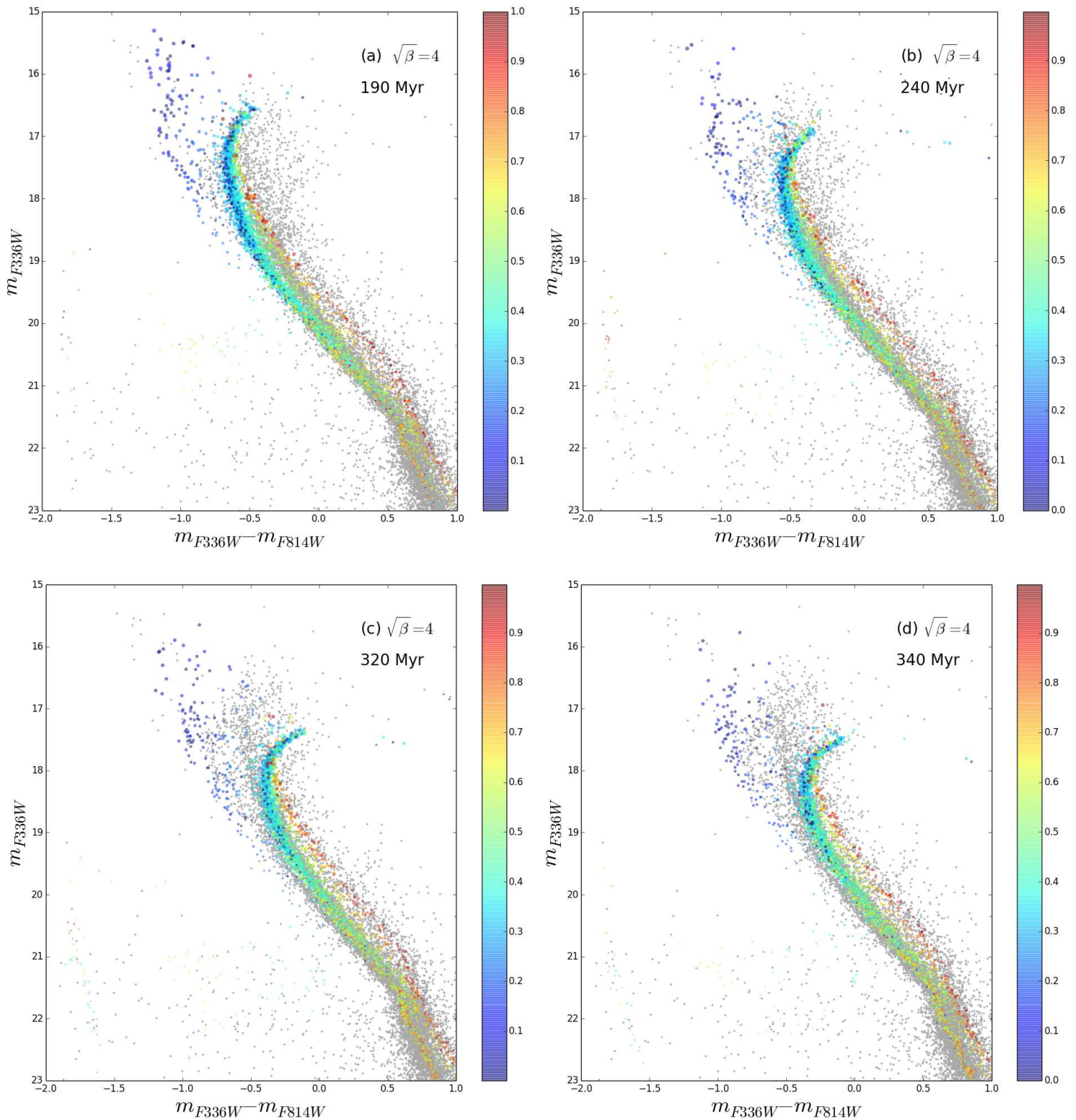

Fig. 6. - Same as Figure 4, but for the populations with different ages. There are approximately 3000 simulated objects in panels $a$ and $b$ and about 2800 simulated objects in panels $c$ and $d$. The simulated stars in the lower-left corners are mainly He-star + white dwarf systems. 

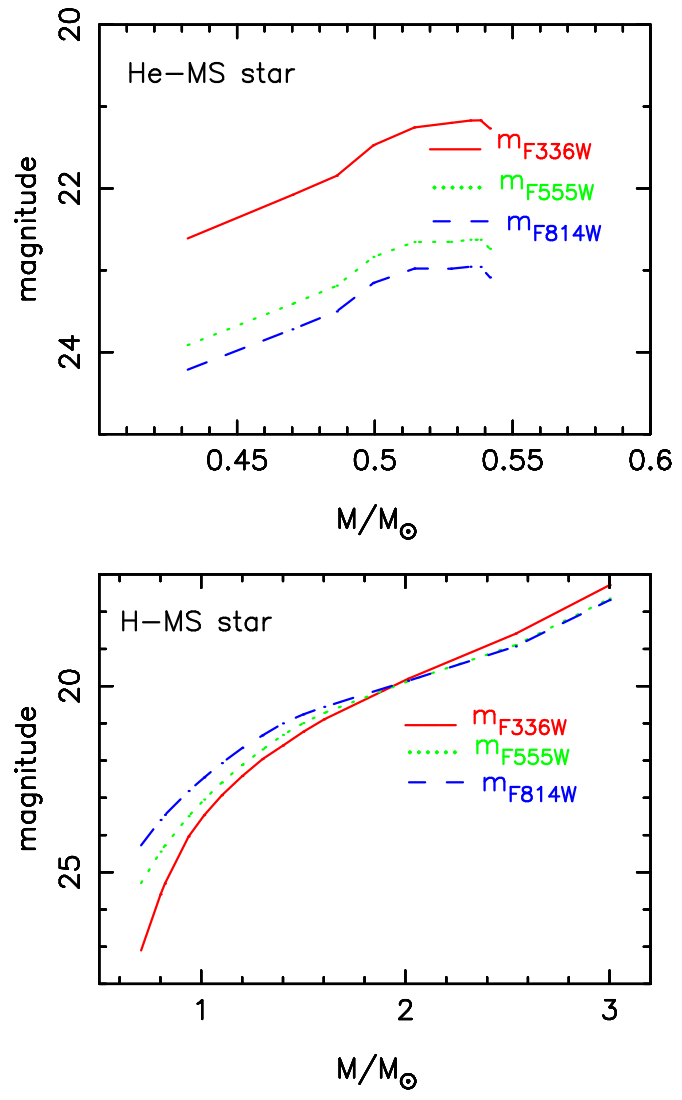

Fig. 7.- The distributions of magnitudes of stars with the age of $340 \mathrm{Myr}$ as a function of mass.
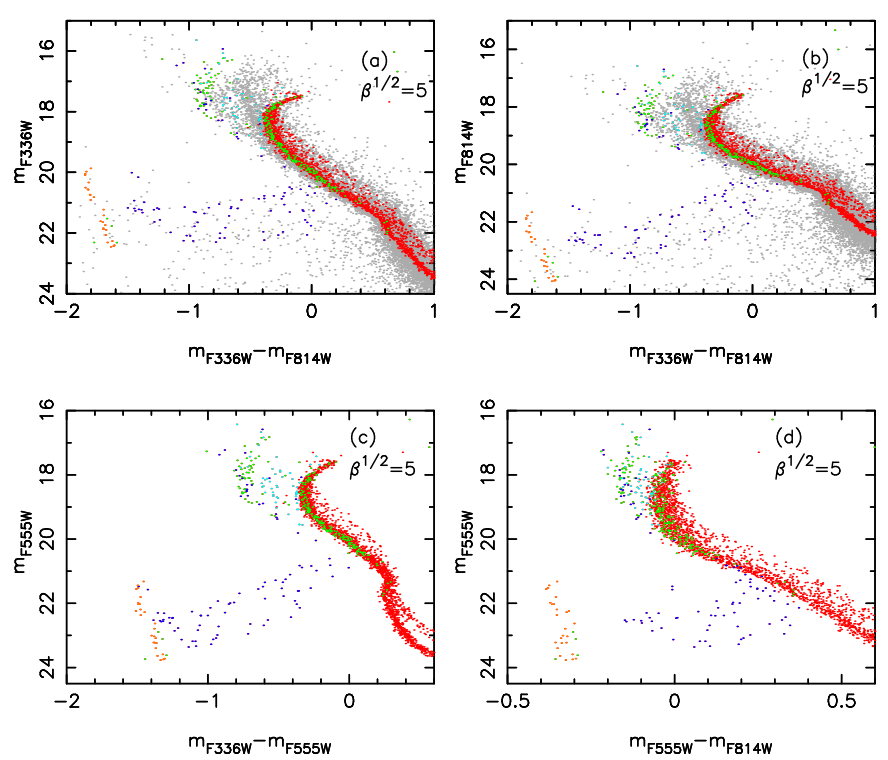

Fig. 8.- CMDs of a simulated population with the age of 340 Myr. There are about 2300 simulated objects in each panel. The merged stars (green dots) make up about 13\%. The four green dots in the lower-left corners of the panels are merged He stars. Other green dots are H-MS stars. The blue and the orange dots represent H-MS + He-star and WD + He-star systems, respectively. The cyan dots denote H-MS + WD systems. 

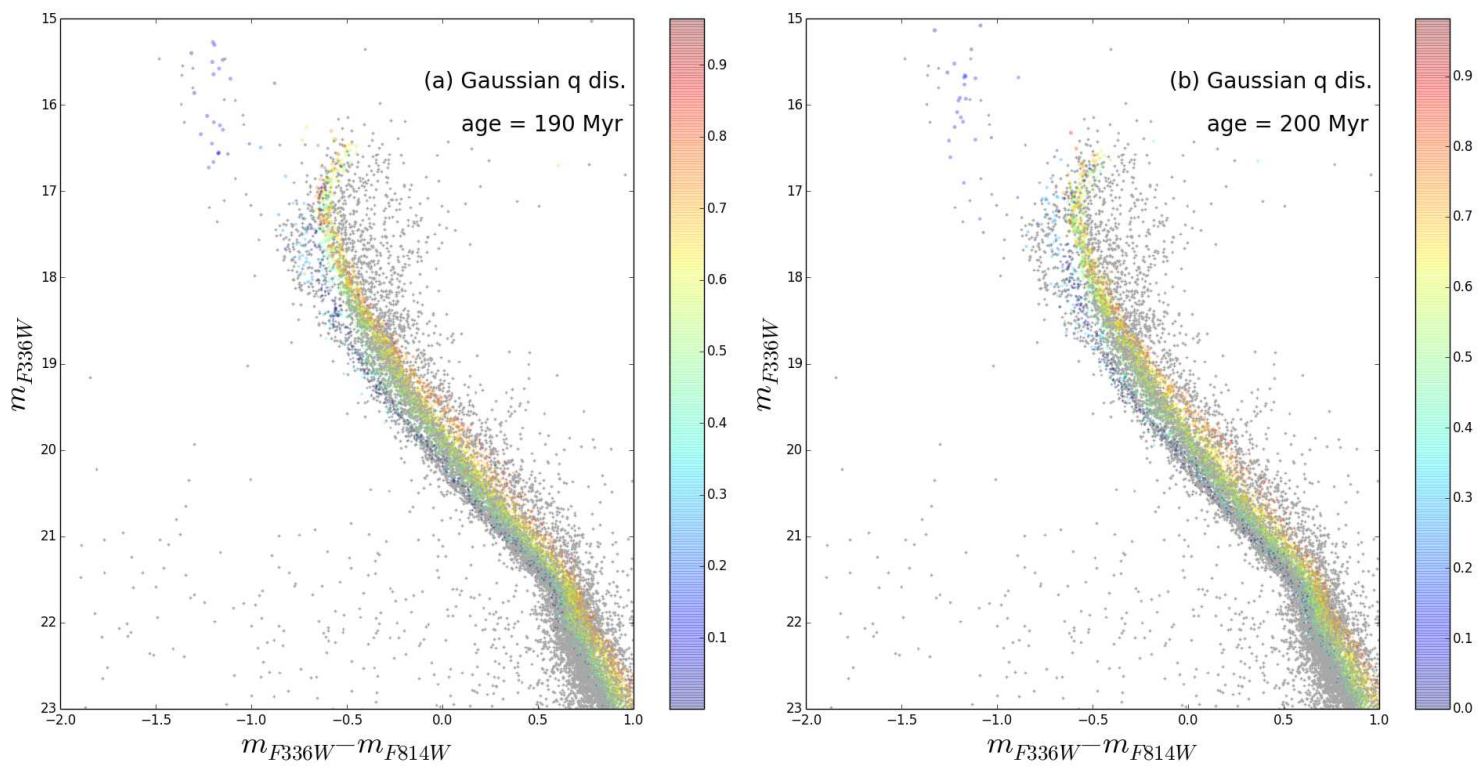

Fig. 9. - Same to Figure 2, but for the population with the Gaussian $q$ distribution. There are about 4000 simulated objects in each panel. The merged stars make up about $12 \%$. Others are binaries. 

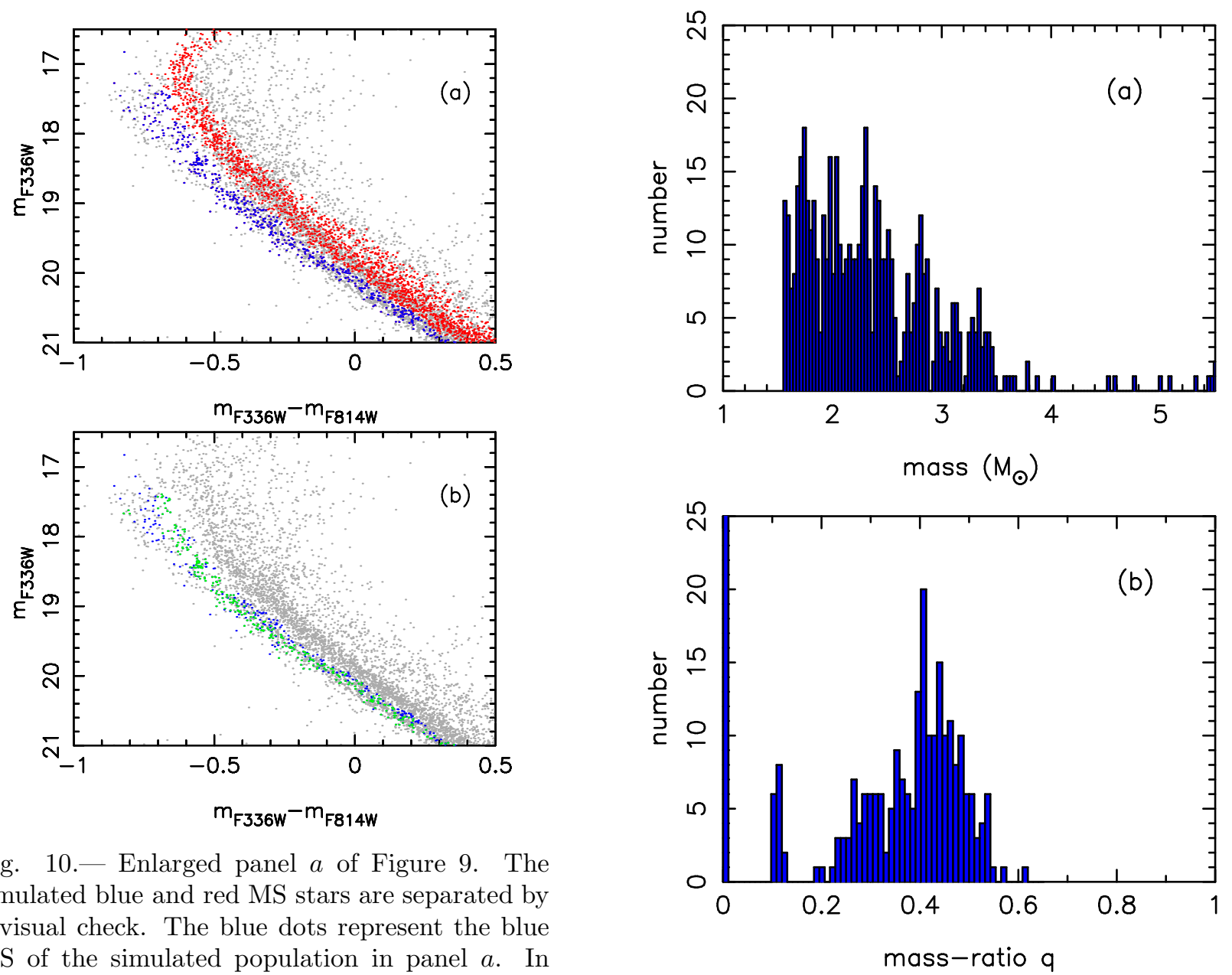

Fig. 11.- Histograms of mass and mass-ratio $q$ of the simulated blue MS stars shown in panel $a$ of Figure 10 . 

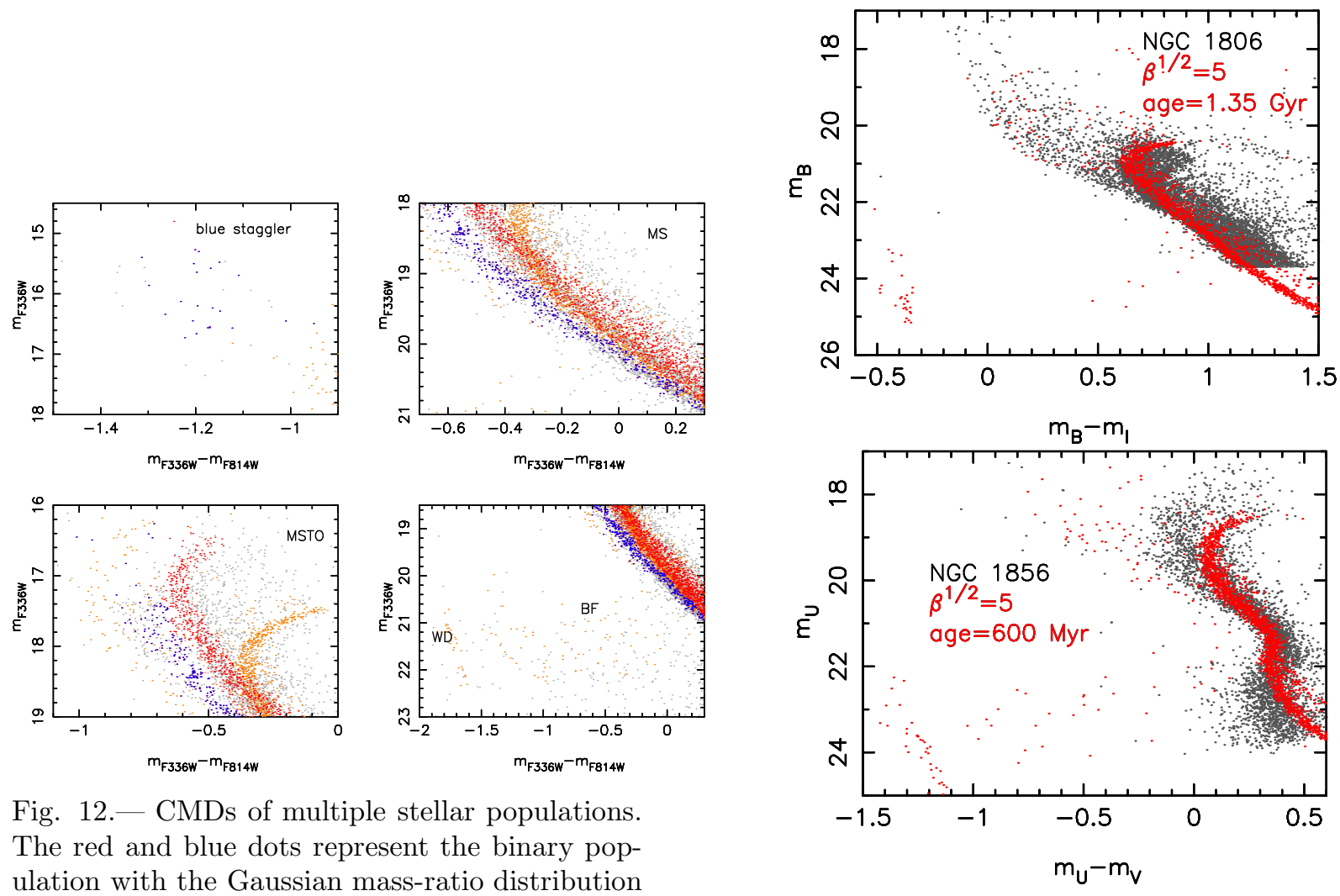

Fig. 12.- CMDs of multiple stellar populations. The red and blue dots represent the binary population with the Gaussian mass-ratio distribution and with the age of $190 \mathrm{Myr}$, while the orange dots shows the population with $\sqrt{\beta}=5$ and with the age of 340 Myr.

Fig. 13.- CMDs of the observed and simulated stellar populations. The dark gray dots and the red ones represent the observed and the simulated stars, respectively. There are about 2000 simulated objects in each panel. The values of distance modulus and reddening $E(B-V)$ are 18.8 and 0.16 for NGC 1806 and 18.5 and 0.08 for NGC 1856, respectively. 


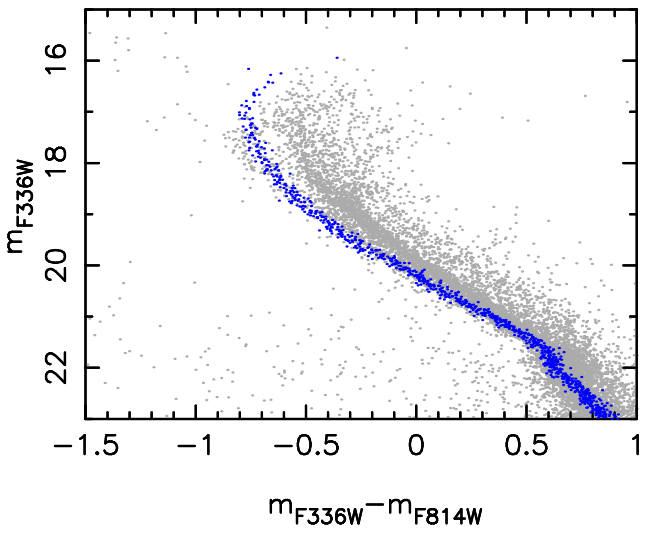

Fig. 14.- The CMD of the simulated SSP (blue dots) with the age of $150 \mathrm{Myr}$.
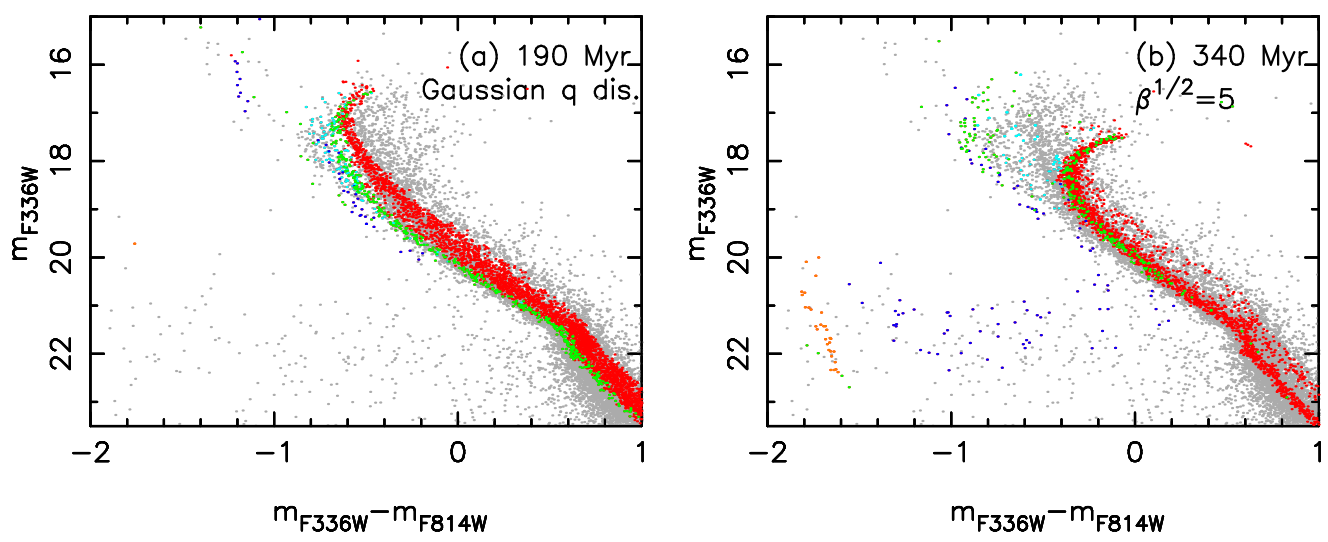

Fig. 15. - CMDs of stellar populations obtained from Salpeter (1955) IMF rather than Chabrier (2001) IMF. The merged stars (green dots) make up around 14\%; others are binaries. The blue and the orange dots represent H-MS + He-star and WD + He-star systems, respectively. The cyan dots denote H-MS + WD systems. 

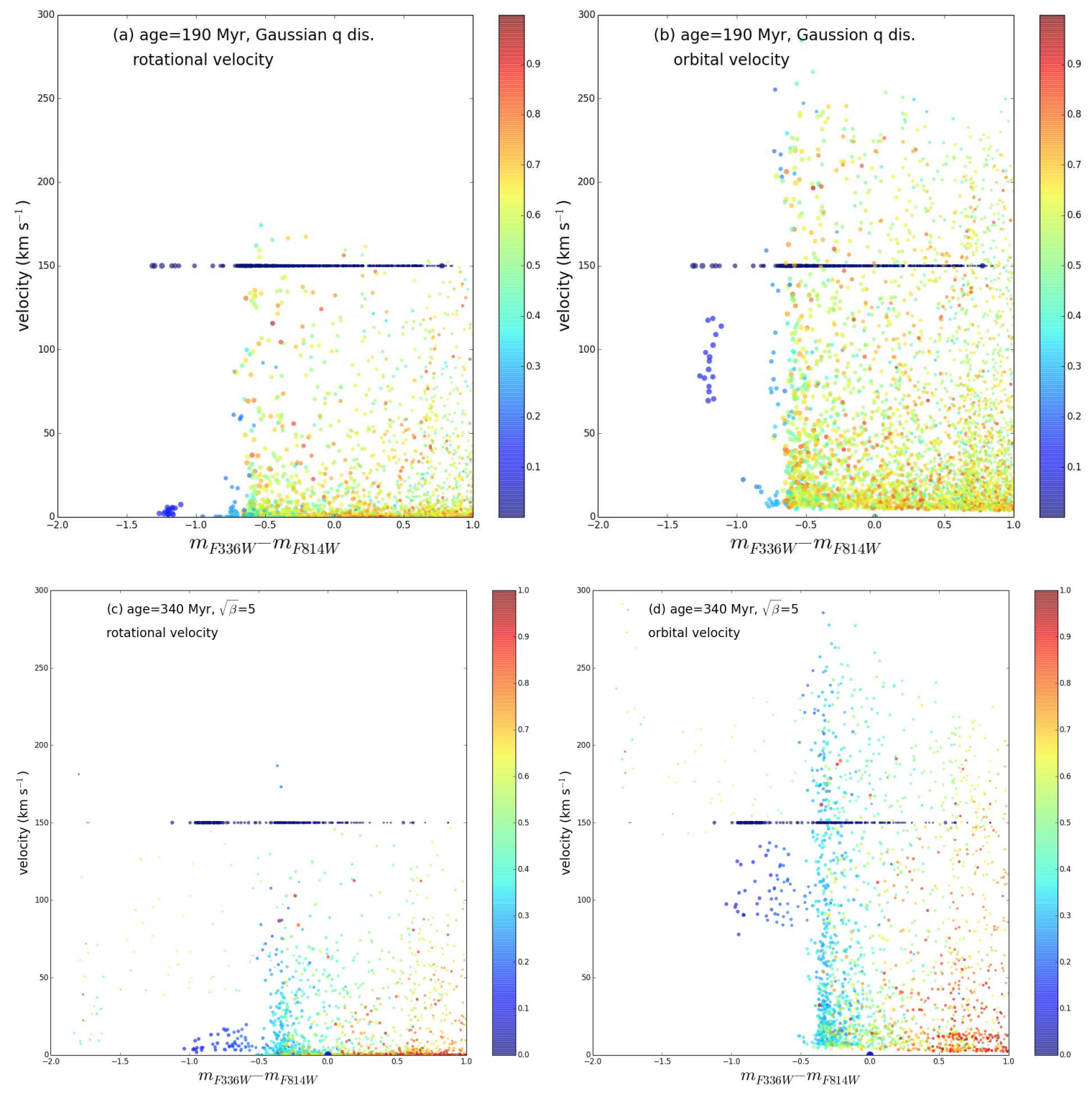

Fig. 16. - Rotational velocities and orbital velocities of simulated populations as a function of $m_{F 336 W}-m_{F 814 W}$. The size and the color of the dots are proportional to stellar mass and massratio $q$, respectively. The velocities of merged stars are assumed to be $150 \mathrm{~km} \mathrm{~s}^{-1}$. 

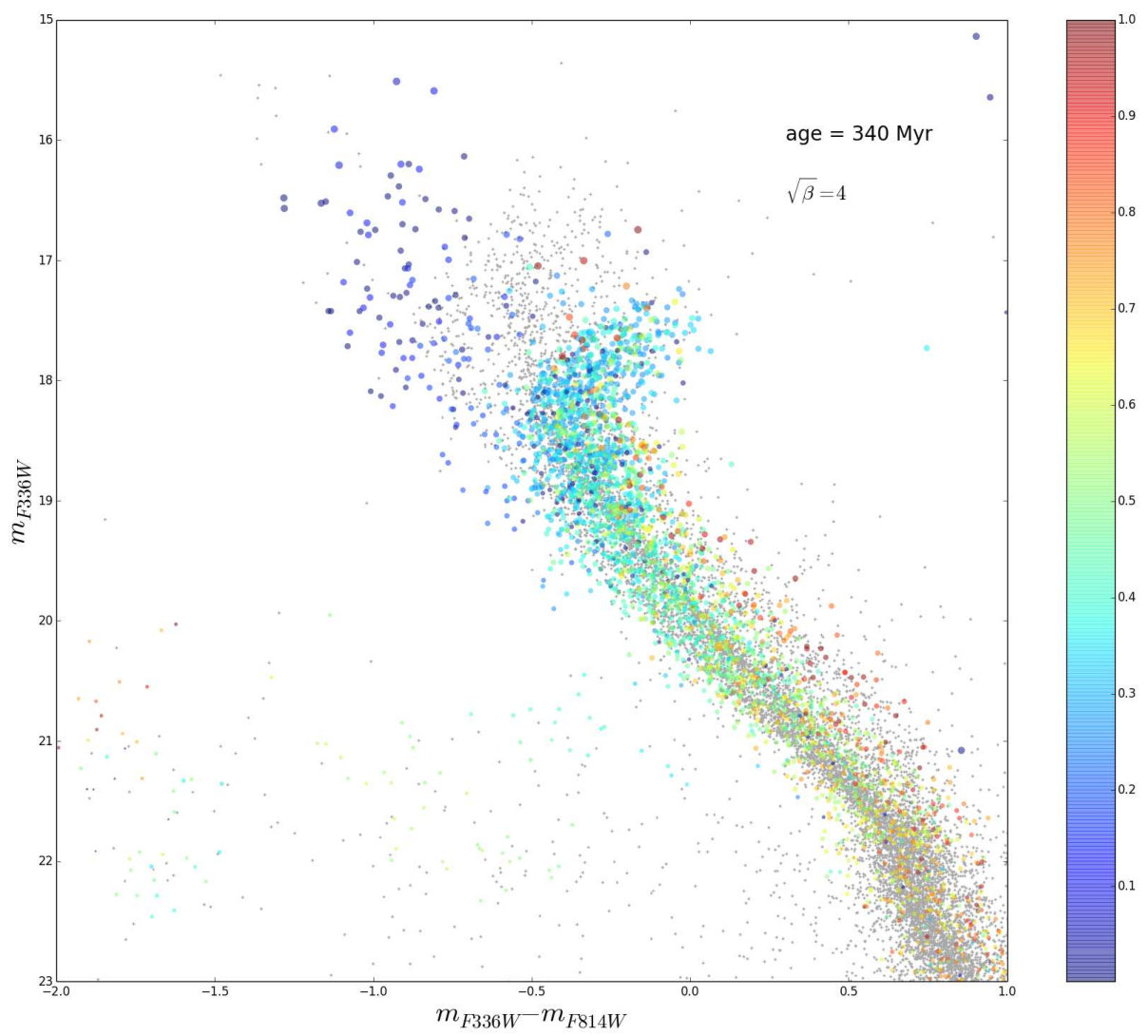

Fig. 17.- Same as Figure 4, but photometric errors for artificial stars are taken to be a Gaussian distribution with a mean value of 0 and a standard deviation of $0.1 \mathrm{mag}$ in magnitude and color. 